\title{
Schach der Gesundheit!
}

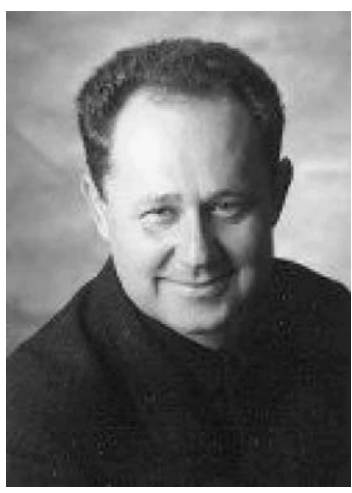

Prof. Dr. Wildner

Bibliografie

DOI 10.1055/s-0029-1225622

Gesundheitswesen 2009;

71: 383-384

(c) Georg Thieme Verlag KG

Stuttgart · New York

ISSN 0941-3790

Korrespondenzadresse

Prof. Dr. M. Wildner

Bayerisches Landesamt für

Gesundheit und Lebens-

mittelsicherheit

Veterinärstraße 2

85762 Oberschleißheim

Manfred.Wildner@Igl.bayern.de
Die im Indien des 4. Jahrhunderts n. Chr. angesiedelte Legende ist jedem Schachspieler vertraut: Um den tyrannischen Herrscher Shihram auf seine Fehler hinzuweisen, erfand der weise Brahmane Sissa ein Spiel, in dem die wichtigste Figur, der König, ohne Hilfe anderer Figuren machtlos ist. Der Unterricht Shihrams im Schachspiel hat den erhofften Eindruck hinterlassen. Um sich für die Vermittlung dieses Stückes Herrschaftswissens zu bedanken, gewährte der König dem Brahmanen einen Wunsch. Scheinbar bescheiden äußerte dieser eine Bitte um Weizenkörner: Auf das erste Feld eines Schachbretts sollte ein Korn gelegt werden, auf das zweite Feld zwei, auf das dritte wiederum die doppelte Anzahl und so weiter. Sissa musste sich zunächst dafür auslachen lassen. Allerdings erfuhr der König nach mehreren Tagen Rechenarbeit seines Rechenmeisters, dass die Menge Getreidekörner im ganzen Reich nicht aufzubringen sei. Historisch interessant ist die Geschichte auch deswegen, weil der mittelalterliche Überlieferer dieser Legende, der Dichter Al-Sabhadi aus Bagdad, die richtige Lösung angegeben haben soll [1]: $2^{64}$ minus 1 Korn oder 18.446.744.073.709.551.615 Weizenkörner. Eine Zahl in Trillionenhöhe. Was die Verlegenheit des Herrschers betraf: Der Rechenmeister half ihm, indem er dazu riet, Sissa das Getreide Korn für Korn abzählen zu lassen.

Der Bezug zu öffentlicher Gesundheit? Mathematisch belegt diese Geschichte eindrucksvoll die Macht der Exponenzialfunktion, in diesem Fall zur Basis 2 (Verdoppelung der Weizenkörner). Sie lässt sich im Bereich der Bevölkerungsgesundheit durch den epidemiologischen Verlauf von Infektionskrankheiten veranschaulichen, z. B. einer Grippepandemie. Wiederum beginnt die Geschichte mit einem ersten Fall, dem Indexfall. Abhängig von der Basisreproduktionszahl $\mathrm{R}_{0}$ sind bei weiteren Ansteckungen dann 2 Sekundärfälle zu beobachten, 4 Tertiärfälle, 8 Fälle der vierten Generation usw. Für die Bevölkerungszahl Deutschlands, etwa 82 Millionen Menschen, sind keine 65 Schachfelder vonnöten: die Grippe-typische Infektion von ca. 1/3 der Bevölkerung ist ohne Gegenmaßnahmen nach spätestens $25 \mathrm{Ge}$ nerationszyklen erreicht. Schach der Gesundheit! Diese Exponenzialfunktion gilt mit Modifikationen auch für die Anfangsphase der Neuen Grippe A/H1N1v. Die Basisreproduktionszahl - sie entspricht dem jeweils Vielfachen an Weizenkörnern - wurde mit Werten von 1,4 bis 3,1 geschätzt [2,3]. Für Japan wurde für Kinder und Jugendliche (Reproduktionszahl 2,8) ein höherer Wert als für die Gesamtbevölkerung (Reproduktionszahl 2,0) berechnet [4]. Die Generationszeit
(GT) ist dabei relativ unbestimmt: sie wird mit etwa 2 Tagen angegeben, aber auch mit 8 bzw. unrealistischen 17 Tagen [2-4]. Die erste Million an Fällen wäre bei einer Basisreproduktionszahl von 2 etwa 20 Generationen nach dem Indexfall erreicht. Abhängig von der Generationszeit wären das 40 Tage (GT 2 Tage) oder auch 160 Tage (GT 8 Tage).

Gegenmaßnahmen der Infektionskontrolle? Ansatzpunkt ist wiederum die Reproduktionszahl R. Diese ist nämlich nur zum Teil von den Eigenschaften des Erregers bestimmt. Zu erheblichen Teilen ist sie auch beeinflusst von der Widerstandskraft der menschlichen Wirte, dem sozialen Kontaktverhalten und gesellschaftlichen Gegebenheiten. Die höhere Reproduktionszahl bei Kindern und Jugendlichen ist zum Teil durch die geringere Kreuzimmunität bestimmt, d.h. Kinder haben immunologisch weniger „Grippeerfahrung“ als ältere Menschen. Zum Teil erklärt sich diese höhere Reproduktionszahl durch intensivere körperliche Kontakte: Die Erforschung der Umwelt auch mit dem Geschmackssinn, das Zusammenstecken der Köpfe beim Spiel, beim Rangeln im Pausehof oder auch später bei den ersten Erfahrungen mit dem ach so spannenden anderen Geschlecht. Hinzu kommen psychologische Unterschiede der Risikowahrnehmung. Als weitere Einflussgröße spielt die Dauer der wirksamen Infektiösität eines Falles innerhalb einer Population eine Rolle.

Aus den genannten Faktoren ergeben sich Optionen für die „Schachzüge“ des bevölkerungsbezogenen Gesundheitsschutzes:

- die rasche Isolierung Erkrankter und die Absonderung erkrankungsverdächtiger Kontaktpersonen durch Einführung einer Meldepflicht, dadurch ausgelöste Maßnahmen des Öffentlichen Gesundheitsdienstes (ÖGD), die Ein- und Ausreiseüberwachung, die Option der Absage von Veranstaltungen und SchlieBung von Einrichtungen,

- die Bevorratung notwendiger antiviraler Medikamente zur Behandlung der Erkrankten und soweit evidenzbasiert sinnvoll zur postexpositionellen Frühbehandlung zur Verkürzung einer möglichen Infektiösität; die Entwicklung, Produktion, Beschaffung und effektive und zeitnahe Applikation von wirksamen und sicheren Impfstoffen,

- die nachhaltige, d.h. effektive und andauernde Information der Bevölkerung zum Sachstand, zur Risikobewertung und zu erwarteten Verläufen sowie zur Propagierung von Verhaltensetiketten (z.B. in-den-Ärmel-Husten/Niesen und häufiges Händewaschen, siehe auch www. wir-gegen-viren.de), 
- eine ebenfalls nachhaltige multidirektionale Kommunikation zwischen den Akteuren (Öffentlicher Gesundheitsdienst, Patientenversorgung im ambulanten und im stationären Bereich), sowohl in Bezug auf das Meldewesen als auch in Bezug auf Leitlinien für die Diagnostik, die Therapie, das Hygieneund Umfeldmanagement einschließlich des verantwortlichen Arbeitsschutzes und die Kommunikation mit Patienten, Angehörigen und der Öffentlichkeit und

- ein in seinen personellen und sachlichen Kapazitäten ausreichend ausgestatteter Öffentlicher Gesundheitsdienst mit den Funktionen Meldewesen, Diagnostik, Infektionskontrolle und Hygienemanagement vor Ort, Erarbeitung von Empfehlungen und Risiko- bzw. Krisenkommunikation.

Berichte aus den USA zeigen, dass diese Optionen insbesondere aufgrund von fehlenden Kapazitäten und fehlenden fortgeschrittenen Qualifikationen im Öffentlichen Gesundheitsdienst nur unvollkommen umgesetzt werden [5]. Vorausschauende Politik sollte dies jedoch leisten. Sie kann neben Kapazitäten auch fachliche Qualifikationen z.B. durch den Auf- und Ausbau im Hochschulbereich („Schools of Public Health“) fördern. Derartige Strukturen sind unverzichtbar, um notwendige Qualifikation für den Umgang mit Unsicherheiten bei Krisen der öffentlichen Gesundheit zu schaffen und vorhandene Kapazitäten zielgerichtet, effektiv und effizient einsetzen zu können [6].

Wie die Forschungsherausforderungen am konkreten Beispiel der Influenza-Pandemie aussehen? Von Epidemiologen der universitären Public Health-Institutionen von Harvard, Hong Kong und London werden genannt [7]: Untersuchungen zur zuverlässigeren Feststellung der Krankheitsschwere auf Ebene des Individuums und auf Ebene der Bevölkerungsgesundheit, Etablierung von Surveillancesystemen, welche eine gegenseitige Kalibrierung erlauben, Stärkung der staatlichen Public Health-Kapazitäten, anhaltende Surveillance der pathogenen Eigenschaften eines neuen Pandemievirus im Verlauf seiner Ausbreitung, ergänzende seroepidemiologische Surveys für eine wissensbasierte Priorisierung von Bevölkerungsgruppen für Impfungen angesichts zumindest zeitweise knapper Impfstoffvorräte, detaillierte Ausbruchsuntersuchungen einzelner Herde, internationale und intersektorale Kooperation zwischen und Koordination von vorhandenen Kompetenzen, abgewogene und abgestimmte Bewertungen durch die damit beauftragten internationalen Organisationen und ethische Aspekte der Verteilungsgerechtigkeit knapper Ressourcen auch bei zwischenstaatlicher Betrachtung.

Lässt sich dadurch eine Pandemie sicher verhindern? Sicher nicht in kurzfristiger (Krisen-)Reaktion - die Neue Grippe A/H1N1v hat die Stufe einer möglichen Verhinderung vermutlich bereits hinter sich [8]. Was kurzfristig möglich ist und vom ÖGD mit seinen vorhandenen Institutionen auf europäischer, nationaler und regionaler Ebene auch effektiv geleistet wird, ist eine Eindämmung und Verzögerung der Infektionsausbreitung. Dies gewinnt Zeit für die Impfungen und die dafür auch benötigten organisatorischen und steuernden Leistungen mit dem Ziele einer in der Sache und aus gesellschaftlicher Sicht zeitigen und gerechten Verteilung [9]. Die Anstrengungen des Bundes, zur europäischen Ebene passende Strukturen z.B. im Rahmen des Robert Koch-Instituts zu schaffen und sich auch an den europäischen Strukturen zu beteiligen, z.B. am Europäischen Zentrum für die Prävention und die Kontrolle von Krankheiten (ECDC) in Stockholm und an infektionsepidemiologischen Trainee-Programmen (z. B. EPIET), haben Früchte getragen. Auch auf Ebene der Bundesländer wurden und werden erfolgreiche Anstrengungen, z.B. durch den Auf- und Ausbau von Landesämtern, unternommen. Die universitären Kooperationen in der Aus-, Fort- und Weiterbildung und die Kapazität in der Fläche sind dabei noch immer entwicklungsbedürftig. An dieser Stelle mag an ein anderes Bild vom Weizenkorn erinnert werden: nämlich das der Aussaat von Weizenkörnern (=Investition) in der Hoffnung auf mittelfristig biblische „100fache, 60fache oder 30fache“ Ernte (=Return on Investment): eine andere Erzählung mit Exponentialfunktion (Matth. 13,23). Diese Investition in dem ÖGD ist geboten, um auf bekannte oder neue Bedrohungen vorbereitet zu sein. Neben der Kapazität zur Krisenreaktion sei daran erinnert, dass Public Health-Maßnahmen täglich von Gesunden in Anspruch genommen werden: in Form der Trinkwasser- und Badewasserüberwachung, umweltmedizinischer Maßnahmen, der Arzneimittelüberwachung, der Tabak- und Suchtprävention u.v.a.m. Gesundheitsschutz bezogen auch auf nicht-infektiöse Krankheiten ist der Versuch einer aufgeklärten Politik, Gesundheitsrisiken vorausschauend zu beherrschen. Eine Einengung des Blickfelds auf kurzfristige Krisenreaktion zu Lasten mittelfristiger Maßnahmen ist dabei eine Versuchung, der gute Führung entschieden entgegen treten sollte. So einfach ist der Schutz der öffentlichen Gesundheit nicht zu erreichen. Um im Bild des Schachspiels zu bleiben: Eine gute Schachstrategie behält das gesamte Spielfeld im Auge, nicht nur eine aktuell bewegte Figur.

In dieser Hoffnung auf gute Investition der Zeit unserer Leser ist das vorliegende Heft auch außerhalb der Infektionsepidemiologie tätig: mit Beiträgen über Inanspruchnahmegradienten in der Schwangerenvorsorge und Risikoverhalten in der Schwangerschaft, über die innere Nickelbelastung dermatologischer Patientinnen und die psychosoziale Versorgungssituation in der ambulanten Akne-Behandlung, über die hausarztzentrierte Versorgung und Einflussfaktoren der Hausbesuchshäufigkeit und mit dem zweiten Teil der Leitlinien zur sozialmedizinischen Beurteilung der Leistungsfähigkeit bei chronischen nicht-malignen Leber- und Gallenwegskrankheiten.

\section{Literatur}

1 Ifrah G. Universalgeschichte der Zahlen. Campus Verlag, Frankfurt/ Main, New York; 1986; S482-S485

2 Fraser C, Donnelly CA, Cauchelmes $S$ et al. Pandemic potential of a strain of influenza A (H1N1): early findings. Published 11 May 2009 on Science Express, DOI: 0.1126/science.1176062 URL: http://www.sciencemag.org/cgi/content/abstract/1176062 (Zugriff 12.06.2009)

3 Boëlle PY, Bernillon P, Desenclos JC. A preliminary estimation of the reproduction ratio for new influenza $\mathrm{A}(\mathrm{H} 1 \mathrm{~N} 1)$ from the outbreak in Mexico, March-April 2009. Euro Surveill 2009; 14 (19): p11=19205 URL: http://www.eurosurveillance.org/ViewArticle.aspx?ArticleId=19205 (Zugriff am 07.06.2009)

4 Nishiura H, Castillo-Chavez C, Safan $M$ et al. Transmission potential of the new influenza $\mathrm{A}(\mathrm{H} 1 \mathrm{~N} 1)$ virus and its age-specificity in Japan. Euro Surveill 2009; 14 (22): pii=19227 URL: http://www.eurosurveillance. org/ViewArticle.aspx?Articleld=19227 (Zugriff am 06.06.2009)

5 Robert Wood Johnson Foundation. Pandemic flu preparedness: lessons from the frontlines June 2009, URL: http://healthyamericans.org/assets/files/pandemic-flu-lesson.pdf (Zugriff am 06.06.2009)

6 Locher W, Wildner M, Kerscher GF. Veränderung gestalten. Der Öffentliche Gesundheitsdienst im internationalen Vergleich - Euregio Bodensee. Zuckschwert, München; 2009

7 Lipsitch M, Riley S, Cauchemez S et al. Managing and reducing uncertainty in an emerging influenza pandemic. N Engl J Med 2009; 10.1056/ NEJMp0904380 updated 28. Mai 2009 URL: http://content.nejm.org/ cgi/content/full/NEJMp0904380 (Zugriff am 07.06.2009)

8 Novel Swine-Origin Influenza A (H1N1) Virus Investigation Team. Emergence of a novel swine-origin influenza A (H1N1) virus in humans. $\mathrm{N}$ Engl J Med 2009; 360: 2605-2615

9 An der Heiden M. Möglicher Verlauf einer Epidemie durch das Neue Influenzavirus A/H1N1 in Deutschland und Auswirkungen präventiver Maßnahmen des Öffentlichen Gesundheitsdienstes. Epidemiol Bull 2009; 22: 219-223 